\title{
An Audit of Staphylococcus aureus Bacteraemia Treatment in a UK District General Hospital
}

\author{
Christine Scarsbrook ${ }^{1}$, Nadeem Sajjad Raja ${ }^{2}$, Michael Hardman ${ }^{3}$, Kath Hughes ${ }^{4}$ \\ ${ }^{1}$ Department of Orthopaedics, Colchester General Hospital, Essex, UK \\ ${ }^{2}$ Department of Clinical Microbiology and Infection Control, Essex, UK \\ ${ }^{3}$ Department of Emergency Medicine, Causeway Hospital, Northern Health and Social Care Trust, Essex, UK \\ ${ }^{4}$ East Sussex Healthcare Trust, Hastings, Essex, UK
}

\begin{abstract}
Objective: Recent guidelines from Healthcare Improvement Scotland recommend that uncomplicated Staphylococcus aureus bacteraemia (SAB) be treated with effective antibiotics for at least 14 days. This audit aimed to see whether Medway Hospital, UK, was following these guidelines, and whether a simple intervention could improve compliance and therefore treatment.
\end{abstract}

Method: All patients with SAB between April 2013 and September 2014 were identified and their clinical notes, laboratory findings and drug charts were reviewed. Starting in January 2014 all SAB were reported on the online pathology results system with the following advice; 'Staphylococcus aureus bacteraemia warrants a minimum of 14 days treatment. Treatment may be longer in cases of deep seated infection.' Outcome measures included 14 days antimicrobial treatment readmission rate with SAB, 3 month mortality post treatment and whether echocardiography was performed.

Results: The demographics of all patients (35 pre and 39 post intervention) were comparable between groups (average age 63 vs $56,54.3 \%$ vs $69.2 \%$ male) although the sources of bacteraemia differed slightly. More patients had indwelling lines in 2013 and there were a higher proportion of IV drug users in 2014; all other risk factors were similar between groups. More patients received appropriate antibiotics post intervention (74\% vs $49 \%$ ). Three month mortality with community acquired $S A B$ increased but mortality with hospital acquired $S A B$ decreased post intervention. Echocardiography uptake improved, and readmission rates with SAB decreased.

Conclusion: The introduction of guidance with blood culture results has demonstrated better compliance with a 14 day minimum treatment length for SAB. Although sample size limits obtaining a statistically significant difference in readmission and mortality rates, this intervention still has the potential to improve treatment of SAB in aiding clinicians to follow guidelines. J Microbiol Infect Dis 2017; 7(4):188-193

Keywords: HIV, HCV, Genotype, Co-infection, Viral load

\section{INTRODUCTION}

Staphylococcus aureus bacteraemia (SAB) is one of the most common serious bacterial infections worldwide with a mortality of $20-30 \%$ and a significant morbidity [1-3]. In 2014, in the UK alone, there were 9,100 cases of SAB reported in Public Health England's voluntary surveillance database, [4] an increase of $1.7 \%$ from the previous year, making the understanding of the optimum treatment of this infection increasingly significant.

Prompt effective treatment and sufficient investigations can help reduce this pathogen's devastating effects. A review of the literature reveals several different strategies used across the globe to improve morbidity and mortality, including investigating and treating affected individuals for infectious endocarditis, involvement of infectious diseases specialists in

Correspondence: Dr Nadeem Sajjad Raja, Department of Clinical Microbiology, The Conquest Hospital, East Sussex Healthcare Trust, Hastings, TN37 7RD, United Kingdom 
care, and administering appropriate antibiotics for a sufficient length of time $[1-3,5,6]$.

Patients with $S A B$ are at a high risk of developing infective endocarditis, a condition associated with higher morbidity and mortality, which requires longer courses of antibiotic treatment. A recent review of nine papers, including a total of 4050 patients with SAB, found $2-15 \%$ patients with $S A B$ had infective endocarditis identified on transthoracic echocardiogram, and $14-28 \%$ identified on transoesophageal echocardiogram [5]. They concluded that all patients with $S A B$ should undergo echocardiogram, with suggestions for which patients would benefit from transoesophageal echocardiogram.

Several studies have shown improved outcomes in patients who had input from infectious diseases specialists. One of these studies, looking at 240 patients with SAB, showed a decrease in mortality in those with infectious diseases consultation, [6,7] and another study of 244 patients, demonstrated a statistically significant reduction in relapse of $S A B$ when clinicians followed microbiologist advice [8].

Health Improvement Scotland issued guidelines in January 2013, recommending that SAB be treated with a minimum of two weeks of intravenous antibiotics [9]. This complements UK [10] and American guidelines [11] for 14 days of antibiotics for methicillin resistant Staphylococcus aureus bacteraemia. Many study results support these guidelines; demonstrating improved mortality in patients treated with 14 days of antibiotics for SAB [12], and decreased relapse rates [13]. However, there is not $100 \%$ uptake of guidelines. A recent study of eight UK hospitals showed that $84 \%$ patients received a 14 day minimum treatment period (intravenous and oral antibiotics), and only $66 \%$ patients overall had 14 days of intravenous antibiotics [14].

Antibiotic treatment length therefore became the main focus for our audit, and we tried a new strategy in our hospital to improve compliance with a 14 day antibiotic prescription for uncomplicated $S A B$ and longer treatment duration in cases of deep seated infections due to Staphylococcus aureus, whilst also looking at uptake of echocardiography (ECHO), and documented microbiology consultation.

\section{METHODS}

This retrospective study was conducted at MFT which is a 600-bed teaching hospital, serving the urban and surrounding areas of Medway town, in the southeast of England, UK. It has well established departments of medicine, surgery, orthopaedics, gynaecology and obstetrics, paediatrics, haematology and oncology, and intensive care. The audit was given approval by MFT audit department prior to starting the study.

All patients with SAB between April $1^{\text {st }} 2013$ and September $30^{\text {th }} 2014$ were identified from the pathology information system and their clinical notes, laboratory findings and drug charts were reviewed by two junior doctors and a consultant microbiologist working in the department at the time. Patients who transferred to other hospitals or who died or had treatment withdrawn for palliation within 14 days of diagnosis were excluded from this study as antibiotic treatment lengths were the primary outcome measure.

Starting in January 2014 all SAB cases were reported on the online pathology results system, accessible to all clinicians, with the following advice; 'Staphylococcus aureus bacteraemia warrants a minimum of 14 days treatment. Treatment may be longer in cases of deep seated infection. Please rule out deep seated infections such as endocarditis, osteomyelitis, discitis or abscess.' The antibiotic treatment length was calculated for each patient and comparisons were made between patients admitted before and after this intervention. We looked both at community and hospital acquired $\mathrm{SAB}$; community acquired $\mathrm{SAB}$ was defined as a $S A B$ that occurred within 48 hours of hospital admission [2].

Secondary outcome measures were the presence of documentation of microbiology advice in patient notes, length of admission, readmission rate with $S A B, 3$ month mortality post treatment, and whether echocardiography was carried out. To determine whether the two groups were similar, demographic information, risk factors for $S A B$ and the source of bacteraemia, as well as the inflammatory markers at diagnosis were compared.

\section{Microbiology methods}


During the study period, the blood culture samples received from patients with septicaemia admitted in MFT were reviewed at the microbiology laboratory. The samples were processed by the BacT/ALERT system (bioMérieux Ltd UK), a continuous blood culture monitoring system which detects the growth of microorganisms. Identification of isolated Staphylococcus aureus was done by the conventional methods and antimicrobial sensitivity was carried out according to The British Society of Antimicrobial Chemotherapy. [15]

\section{RESULTS}

During the study period, 100 patients had SAB, 74 of whom fitted the inclusion criteria, 35 pre intervention (2013) and 39 post intervention (2014). Of the 74 cases of SAB, 51 patients presented with community acquired SAB (26 cases of $S A B$ in 2013 and 25 cases in 2014) while only 23 patients acquired $S A B$ during their stay in the hospital (10 patients in 2013 and 13 patients in 2014). The demographics were comparable between both groups (average age 63 vs $56,54.3 \%$ vs $69.2 \%$ male). Average inflammatory markers were lower in the first group than second group (White cell count 12.0 vs $13.3 \times 10^{9} / \mathrm{L}$, C-reactive protein 127.2 vs 161.7 $\mathrm{mg} / \mathrm{L})$.

The most common source of SAB was skin in both groups and least common source was urinary tract infection. Ten patients in this study had line related sepsis; 6 pre intervention and 4 post intervention. The source of sepsis remained unknown in three cases in 2013 and three cases in 2014. Graph 1 illustrates the sources of SAB in 2013 and 2014.

In terms of risk factors for $\mathrm{SAB}$, a higher proportion of patients in 2013 had diabetes $(13 / 35, \quad 37 \%)$, cellulitis (14/35, 40\%), ulcers/wounds $(14 / 35,40 \%)$, malignancy $(10 / 35$, $29 \%$ ) and indwelling lines $(13 / 35,37 \%)$ as compared to patients in 2014, with risk factor rates as follows: diabetes $(11 / 39,28 \%)$, cellulitis (7/39, 18\%), ulcers/wounds (11/39, 28\%), malignancy $(6 / 39,15 \%)$ and indwelling lines $(7 / 39,18 \%)$. There were a higher proportion of intravenous drug users in 2014, as well as more patients with liver disease in 2014; all other risk factors were very similar between groups. Table
1 shows the common risk factors for $S A B$ in each group.

The length of hospital stay with SAB remained the same during 2013 and 2014. There was an improvement in the proportion of patient notes which contained documentation of microbiology advice post intervention, in 2014 , from $83 \%$ to $90 \%$. There was also a greater uptake of echocardiography to look for evidence of endocarditis in patients in 2014 (16/39, 41\% compared with 10/35, 28\%). (Table2).

Treatment in all patients was initially with intravenous antibiotics, and then some patients were converted to oral antibiotic treatment. More patients received appropriate antibiotics for an adequate treatment period after the intervention $(30 / 39,74 \%)$ compared with beforehand $(17 / 35,49 \%)$ (Table2). In this study, 50 patients (20 in 2013 and 30 in 2014) received at least 14 days of treatment, and 24 patients received fewer than 14 days of treatment (15 in 2013 and 9 in 2014). Average treatment length for uncomplicated SAB in 2014 was 16.7 days, which was better than the 11 day average in 2013. In deep seated SAB the average treatment duration was also higher in 2014 (Table2). The most commonly used antibiotic identified in this audit was flucloxacillin (44 patients) followed by co-amoxiclav, piperacillin/tazobactam, gentamicin and vanomycin, or teicoplanin or linezolid for those with a penicillin allergy.

On the basis of the 74 available patient records, readmission rates with bacteraemia were improved post intervention (5\% (2/35 patients) compared with (5/39 patients) $15 \%$ ). The overall 3 month mortality for all patients with SAB during the time studied was $12 \% \quad(9.10 \% \quad(3 / 35$ patients) in 2013 and 16\% (6/39 patients) in 2014). Compare to the survivors, the mortality was significantly associated with male gender, old age (with a mean age of 66.88 years). Following the intervention in 2014 there was a $58 \%$ improvement in 3 month mortality in those with healthcare associated SAB. However, the mortality rate at 3 months in community acquired $\mathrm{SAB}$ seemed to increase, from 5\% (1/35 patients) in 2013, to $20 \%$ in 2014 (Table2). The following risk factors for mortality were significantly more associated with community acquired $S A B$ than for healthcare associated 
SAB: liver impairment and diabetes. Skin and abdomen were the main source of infection in this group. In contrast mortality was lower in patient with chest source of sepsis and

Table 1. Risk factors for SAB in 2013 and 2014.

\begin{tabular}{lcc}
\hline Risk factors for SAB & $\begin{array}{c}\text { Number of } \\
\text { Patients (\%) in } \\
2013 . \mathrm{n}=35\end{array}$ & $\begin{array}{c}\text { Number of } \\
\text { Patients (\%) in } \\
\text { 2013. } \mathrm{n}=39\end{array}$ \\
\hline Diabetes & $13(37)$ & $11(28)$ \\
Steroids & $4(11)$ & $5(13)$ \\
Cancer & $10(29)$ & $6(15)$ \\
HIV & $0(0)$ & $0(0)$ \\
Renal impairment & $8(23)$ & $9(23)$ \\
Liver disease & $5(14)$ & $10(26)$ \\
Intravenous drug use & $1(3)$ & $5(13)$ \\
Bedbound & $0(0)$ & $1(3)$ \\
Nursing/residential & $6(17)$ & $2(5)$ \\
home & & \\
Indwelling lines & $13(37)$ & $7(18)$ \\
Operative wound & $4(11)$ & $6(15)$ \\
Ulcer/skin lesion & $14(40)$ & $11(28)$ \\
Cellulitis & $14(40)$ & $7(18)$ \\
Prosthetic material & $3(9)$ & $4(10)$ \\
\hline
\end{tabular}

undergoing intravascular device removal as well as Intensive Therapy Unit admission.

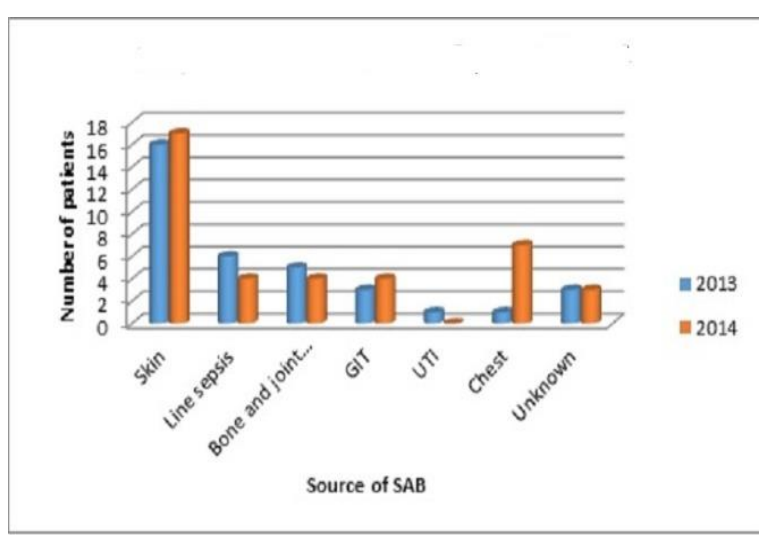

GIT: Gastrointestinal tract, UTI: Urinary tract infection Figure 1. Source of in 2013 and 2014.

Table 2. Outcome measures in SAB audit

\begin{tabular}{lcc}
\hline Variables & $\mathbf{2 0 1 3}(\mathbf{N}=\mathbf{3 5})$ & $\mathbf{2 0 1 4}, \mathbf{N}=\mathbf{3 9}$ \\
\hline Appropriate antibiotic and length of treatment & 17 patients (49\%) & $\mathbf{3 0}$ patients (74\%) \\
Average treatment length (excluding deep-seated infections) & 11 days & 16.7 days \\
Average treatment length (discitis, osteomyelitis, septic arthritis) & 44.4 days & 46.9 days \\
Documented microbiology discussion in notes & 29 patients (83\%) & 16 patients (90\%) \\
Echocardiography performed & 10 patients (28\%) & 16 patients (41\%) \\
Length of admission following SAB diagnosis & 20.5 days & 20.5 days \\
Readmission rates with bacteraemia & 5 patients (15\%) & 2 patients (5\%) \\
Proportion SAB which are healthcare associated & 11 patients (31\%) & 13 patients (33\%) \\
3 month mortality: healthcare associated SAB & 2 patients (18\%) & 1 patient $(8 \%)$ \\
3 month mortality: community acquired SAB & 1 patient (5\%) & 5 patients (20\%) \\
3 month mortality: SAB all cases & 3 patients (9\%) & 6 patients (16\%)
\end{tabular}

\section{DISCUSSION}

Staphylococcus aureus is known to be a major cause of bacteraemia, endocarditis, abscess formation, bone and joint infections as well as recurrent bacteraemia. Staphylococcus aureus infections are associated with significant morbidity and mortality. Inappropriate antimicrobial therapy leads to increased healthcare associated costs, prolonged hospital stay and even increased mortality rates $[12,13)$. Current International and United Kingdom guidelines recommend a minimum of 14 days treatment for uncomplicated bacteraemia and longer treatment such as 4-6 weeks in deep seated infections three [9-11]. These recommendations help to reduce relapse. Our study confirms the reduction of readmission due to $S A B$ relapse after the introduction of the computer based advice to clinicians with positive culture results. The increased compliance $(74.4 \%$ from $49 \%)$ with the antibiotic guidance in the post intervention period correlated with a reduction in mortality in healthcare associated $S A B$ during this period. In all cases of healthcare associated bacteraemia, Staphylococcus aureus did not have a significant effect on the mortality rate. The mortality result in community acquired bacteraemia is influenced by a few patients who died from unrelated conditions such as lung cancer, pre-existing renal disease etc. Mortality was highest in the over 65 age group. In this 
study the overall mortality (12\%) in three months was lower than in a previous study in the literature (24\%) [16].

Another recent study trialled an intervention in which a form comprising six recommendations for Staphylococcus aureus management (including a 14 day minimum antibiotic treatment period) was put in patients' notes and a microbiologist monitored compliance. This intervention did result in a statistically significant reduction in 14-day mortality [17].

A multi-centre cohort study of over 800 patients demonstrated a link between infectious disease specialist consultation and improved uptake of echocardiograms, repeat blood cultures, better compliance with the recommended antibiotic type and treatment length, and reduced inhospital mortality [6]. In this audit, after the introduction of advice added to lab results, the authors also observed improvement in the documentation of the microbiology advice in the patient's notes and found that more patients underwent echocardiography. Due to the limited sample size, and other limitations such as this being a retrospective study, the audit team were unable to assess if the echocardiography results improved any outcome in patients with $\mathrm{SAB}$. Despite overall appropriate antibiotic regimens, the total length of stay during pre and post intervention remained similar.

Several risk factors for developing $S A B$ have been identified in the literature. These risk factors include intravenous drug use, skin lesions or cellulitis, surgical site infection, malignancy, immunosuppression, age, haemodialysis, diabetes mellitus, alcohol excess, congestive cardiac failure, neutropenia, prosthetic material, HIV and intravascular devices. The presence of one or more comorbidities is also associated with higher mortality in patients with SAB $[1,2,6,12,13]$. In our study we identified similar risk factors for $S A B$ such as ulcers/skin lesions, cellulitis, diabetes mellitus, indwelling lines, malignancy, renal impairment and liver disease. We also noted that in our study more than two thirds of $S A B$ cases were acquired in the community. These findings are consistent with a previous study from Australia and New Zealand [18].

Although it was not possible to determine with certainty the source of sepsis in our all patients, skin and soft tissue was found to be the most common source (33 patients in our study). Other common sources were line-related sepsis (10 patients), followed by bone and joint (nine patients), chest (eight patients) and gastrointestinal tract (seven patients). Tong et al from Northern Australia also reported the high incidence of both skin and soft tissue infections and SAB [19].

\section{Conclusion}

This simple intervention appears to have the potential to improve treatment of $S A B$ by increasing clinician awareness of the importance of thorough investigation and appropriate treatment of patients with $S A B$ infections, helping them follow the guidelines to prevent SAB complications. SAB represents a serious infection, associated with serious complications, early especially community acquired $S A B$ and late mortality. This study also highlighted higher mortality in community acquired SAB. This result was influenced in a few patients who died from unrelated comorbidities. The clinicians, including microbiologists, should be more vigilant in treating $S A B$ especially patients with pre-existing conditions as well as community acquired and the deep seated infections caused by Staphylococcus aureus with appropriate antibiotics. By ensuring that all patients receive appropriate investigations and treatment, this should help to prevent SAB complications and thereby reduce strain on health resources.

\section{ACKNOWLEDGMENTS}

Conflict of interest: The authors declare no personal or financial conflict of interest.

Funding disclosure: Non to declare

\section{REFERENCES}

1) Thwaites GE, Edgeworth JD, Gkrania-Klotsas E, et al. UK Clinical Infection Research Group. Clinical Management of Staphylococcus aureus Bacteraemias. Lancet Infect Dis 2011; 11: 208-22.

2) Van Hal SJ, Jenson S, Vaska V, et al. Predictors of Mortality in Staphlococcus aureus Bacteraemia. Clin Microbiol Rev 2012; 25: 362-386.

3) Wyllie D, Crooke D, Peto T. Mortality after Staphylococcus aureus bacteraemia in two hospitals 
in Oxfordshire, 1997-2003: cohort study. BMJ 2006; 333: 281.

4) Health Protection England: Health Protection Report. Voluntary surveillance of Staphylococcus aureus bacteraemia in England, Wales and Northern Ireland: 2007-2014 [online] (2015). Available at: https://www.gov.uk/government/uploads/system/uploa ds/attachment_data/file/455607/hpr2915_saureus.pdf [Accessed 30.04.2016].

5) Holland TL, Arnold C, Fowler VG Jr. Clinical Management of Staphylococcus Bacteremia: A Review. JAMA 2014; 312: 1330-1341.

6) Bai $A D$, Showler $A$, Burry $L$, et al. Impact of Infectious Disease Consultation on Quality of Care, Mortality, and Length of Stay in Staphylococcus aureus Bacteremia: Results From a Large Multicenter Cohort Study. Clin Infect Dis 2015; 60: 1451-1461.

7) Lahey $T$, Shah R, Gittzus J, Schwartzman J, Kirkland K. Infectious diseases consultation lowers mortality from Staphylococcus aureus bacteremia. Medicine (Baltimore); 88: 263-267.

8) Fowler VG Jr, Sanders LL, Sexton DJ, et al. Outcome of Staphylococcus aureus bacteremia according to compliance with recommendations of infectious diseases specialists: experience with 244 patients. Clin Infect Dis 1998; 27: 478.

9) Healthcare Improvement Scotland. Guidance on management of proven or suspected Staphylococcus aureus bacteraemia in adults (2015). [online] Available

at: https://www.scottishmedicines.org.uk/files/sapg1/SAB _algorithm.pdf [Accessed 04.05.2016].

10) Gould FK, Brindle R, Chadwick PR, et al. MRSA Working Party of the British Society for Antimicrobial Chemotherapy. Guidelines (2008) for the prophylaxis and treatment of methicillin-resistant Staphylococcus aureus (MRSA) infections in the United Kingdomp. J Antimicrob Chemother 2009; 63: 849-861.

11) Liu C, Bayer A, Cosgrove SE, et al. Clinical Practice Guidelines by the Infectious Diseases Society of America for the Treatment of MethicillinResistant Staphylococcus aureus Infections in Adults and Children. Clin Infect Dis 2011; 52: 18-55.

12) Jensen $A G$, Wachmann $C H$, Espersen $F$, Scheibel J, Skinhøj P, Frimodt-Møller N. Treatment and outcome of Staphylococcus aureus bacteraemia: a prospective study of 278 cases. Arch Intern Med 2002; 162: 25-32.

13) Chong YP, Moon SM, Bang KM, et al. Treatment duration for uncomplicated Staphylococcus aureus bacteraemia to prevent relapse: analysis of a prospective observational cohort study. Antimicrob Agent Chemother 2013; 57:1150-1156.

14) Thwaites GE; United Kingdom Clinical Infection Research Group (UKCIRG). The management of Staphylococcus aureus bacteremia in the United
Kingdom and Vietnam: a multi-centre evaluation. PLoS One 2010; 13; 5 (12):e14170.

15)EUCAST disk diffusion method. The British Society for Antimicrobial Chemotherapy, (2016). available at: http://bsac.org.uk/eucastbsac-discdiffusion-method/ [Accessed 02.01.2017]

16) Ringberg H, Thoren A, Lilja B. Metastatic complications of Staphlococcus aureus septicaemia. To seek is to find. Infection 2000; 28(3): 132-36.

17) López-Cortés LE, Del Toro MD, Gálvez-Acebal J, et al. REIPI/SAB group. Impact of an evidence-based bundle intervention in the quality-of-care management and outcome of Staphylococcus aureus bacteremia. Clin Infect Dis 2013; 57(9):1225-33.

18) Turnidge JD, Kotsanas $D$, Munckhof $W$, et al. Australia New Zealand Cooperative on Outcomes in Staphylococcal Sepsis. Staphylococcus aureus bacteraemia: a major cause of mortality in Australia and New Zealand. Med J Aust 2009 Oct 5; 191(7):368-373.

19) Tong SY, Bishop EJ, Lilliebridge RA, et al. 2009. Community-associated strains of methicillin-resistant Staphylococcus aureus and methicillin-susceptible S. aureus in indigenous northern Australia: epidemiology and outcomes. J Infect Dis 2009; 199: 1461-1470. 